\title{
Tuning improved anisotropic actions in lattice perturbation theory
}

\author{
Justin Foley* and Colin Morningstar ${ }^{\dagger}$ \\ Department of Physics, Carnegie Mellon University, Pittsburgh, PA 15213 \\ E-mail: jfoleyeandrew. cmu.edu \\ E-mail: colin_morningstarecmu.edu
}

We discuss the tuning of the anisotropic clover action and Symanzik-improved gauge action in lattice perturbation theory. The fermion action is constructed from stout-smeared spatial links, which complicates the calculation considerably. In addition, the full quark-mass dependence of the action parameters is included in this study. We present results for the fermion and gauge aspect ratios for varying bare aspect ratios, quark masses and smearing parameters.

The XXVI International Symposium on Lattice Field Theory

July 14-19 2008

Williamsburg, Virginia, USA

\footnotetext{
* Speaker.

${ }^{\dagger}$ For the Hadron Spectrum Collaboration.
} 


\section{Introduction}

In recent years, anisotropic lattice actions have seen increasing use in simulations. The $3+1$ anisotropic lattice, which has a temporal lattice spacing $a_{t}$ that is much less than the spatial lattice spacing $a_{s}$, is obviously very useful in studies of QCD at non-zero temperature, where it allows the temperature to be varied precisely at fixed lattice spacing. At zero temperature, the utility of the anisotropic lattices in studies of the glueball spectrum of pure Yang-Mills is well-known [1, 2]. The fine temporal lattice spacing helps to resolve higher-lying states whose two-point functions quickly disappear into noise, while the relative coarseness of the spatial lattice spacing minimises the computational overhead.

The ultimate goal of the Hadron Spectrum Collaboration is a precise determination of the low-lying spectrum of QCD. The low energy states of interest are not, however, restricted to just the ground state resonances in each irreducible representation of the lattice symmetry group, but include a number of higher-lying excitations. The use of anisotropic lattices with fine temporal lattice spacings will be essential for the resolution of these states. It is also crucial that the action used should have a well-defined single timeslice transfer operator, to avoid unphysical oscillations in the temporal fall-off of correlation functions. This requirement is potentially at odds with the Symanzik improvement program, which aims to eliminate lattice artifacts in a systematic way by adding irrelevant operators to the lattice action. However, on an anisotropic lattice, cutoff effects which depend on $a_{t}$ are highly suppressed and the dominant lattice artifacts depend on the spatial lattice spacing only. Therefore, a Symanzik-type improvement program can be implemented which removes these dominant cutoff effects, without sacrificing positivity of the action.

The advantages associated with anisotropy come at a price. Anisotropic lattices break hypercubic symmetry, and accordingly the quark and gauge actions contain additional parameters which must be tuned so that the anisotropy (or aspect ratio) $a_{s} / a_{t}$ measured using different physical probes takes a fixed target value. This tuning can be performed non-perturbatively [3]. However, in principle, non-perturbative tuning runs may be required for each new set of simulation parameters. In this proceedings, we describe the tuning of the anisotropic action parameters in one-loop perturbation theory. These lattice perturbative results are valid in the high $\beta$ regime. However, the ultimate goal of this work is to combine the results of lattice perturbation theory with the non-perturbative data to obtain functional forms for the action parameters which hold over much of parameter space.

\section{Actions}

The anisotropic quark action used in our simulations is

$$
\begin{aligned}
S_{\text {quark }}=a_{t} a_{s}^{3} \sum_{x} \bar{\psi}(x) & \left\{m_{0}+\gamma_{t} \nabla_{t}-\frac{a_{t}}{2} \triangle_{t}+v_{s} \sum_{k}\left(\gamma_{k} \nabla_{k}-\frac{a_{s}}{2} \triangle_{k}\right)\right. \\
+ & \left.\frac{1}{2}\left[c_{t} a_{s} \sum_{k} \sigma_{t k} F_{t k}+c_{s} a_{s} \sum_{k<l} \sigma_{k l} F_{k l}\right]\right\} \psi(x),
\end{aligned}
$$

where the covariant derivatives and clover-leaf discretisation of the field strength tensor are built from link variables which have been stout-smeared [4] in the spatial directions. As noted above, 
it is important that the temporal links be left unsmeared. Furthermore, the action includes tadpole improvement factors for the spatial links, although, for an action constructed from smeared links, these are close to unity.

In a numerical simulation all lengths are expressed in lattice units, and the quark action depends on a bare anisotropy parameter which we denote $\xi_{0}$. This bare parameter can receive radiative corrections. However, it is possible to choose the coefficient $v_{s}$, multiplying the kinetic term, such that the aspect ratio measured from the quark dispersion relation takes a predefined target value. At tree level and in the chiral limit, setting $v_{s}=1$ fixes the measured aspect ratio to the bare anisotropy. The 'clover' coefficients $c_{t}$ and $c_{s}$ are tuned such that on-shell quantities are free of $\mathscr{O}\left(a_{t}, a_{s}\right)$ cutoff effects. For massless quarks, their tree-level values are $c_{t}=\frac{1}{2}\left(v_{s}+\frac{1}{\xi_{0}}\right), c_{s}=v_{s}$.

The gauge action incorporates Symanzik and tadpole improvement, and can be written

$$
S_{\text {gauge }}=-\beta\left\{\xi_{0}\left[\frac{4}{3} \sum_{i} P_{t i}-\frac{1}{12} \sum_{i} R_{t i}\right]+\frac{1}{\xi_{0}}\left[\frac{5}{3} \sum_{i<j} P_{i j}-\frac{1}{12} \sum_{i<j}\left(R_{i j}+R_{j i}\right)\right]\right\} .
$$

$R_{i j}$ denotes a $1 \times 2$ rectangle, two links long in the $j$ direction, summed over all lattice sites. The dominant discretisation errors of this action appear at $\mathscr{O}\left(a_{t}^{2}, a_{s}^{4}, \alpha_{s} a_{s}^{2}\right)$. Crucially, the lagrangian is just one link wide in the temporal direction, which guarantees a well-defined single-timeslice lattice transfer operator.

\section{Tuning the quark action parameters}

In lattice perturbative studies, for the sake of simplicity, one often ignores the dependence of the action parameters on the bare quark mass. In the Fermilab formalism [5], on the other hand, the full quark-mass dependence of the action parameters is determined, yielding an action which can be used in both the chiral and heavy-quark regimes. In our simulations, $m_{0} a_{s}$ is not always guaranteed to be very small, and we have adopted a Fermilab-type approach in this study. Even at the tree-level, the parameters of the quark action receive non-trivial mass-dependent corrections. The tree-level mass dependence of the kinetic coefficient $v_{s}$ can be determined by expanding the free-quark energy in powers of the spatial momentum, and demanding that the quark rest mass and kinetic mass be equal for a given aspect ratio:

$$
a_{t} E(\vec{p})=a_{t} M_{\mathrm{rest}}+\frac{1}{2 a_{t} M_{\mathrm{kin}}} \frac{\left|a_{s} \vec{p}\right|^{2}}{\xi_{0}^{2}}+\cdots
$$

This implies that

$$
v_{s}^{(0)}=\sqrt{\frac{1}{4} \xi_{0}^{2} \sinh ^{2}\left(a_{t} M_{\text {rest }}^{(0)}\right)+\exp \left(a_{t} M_{\text {rest }}^{(0)} \frac{\sinh \left(a_{t} M_{\text {rest }}^{(0)}\right)}{a_{t} M_{\text {rest }}^{(0)}}\right.}-\frac{\xi_{0}}{2} \sinh \left(a_{t} M_{\text {rest }}^{(0)}\right),
$$

where $a_{t} M_{\text {rest }}^{(0)}=\log \left(1+a_{t} m_{0}\right)$. Expanding this expression, one finds that the leading order correction to $v_{s}^{(0)}$ is linear in the bare mass. The tree-level chromoelectric and chromomagnetic coefficients can be determined by requiring that, at low momenta, scattering amplitudes match their 


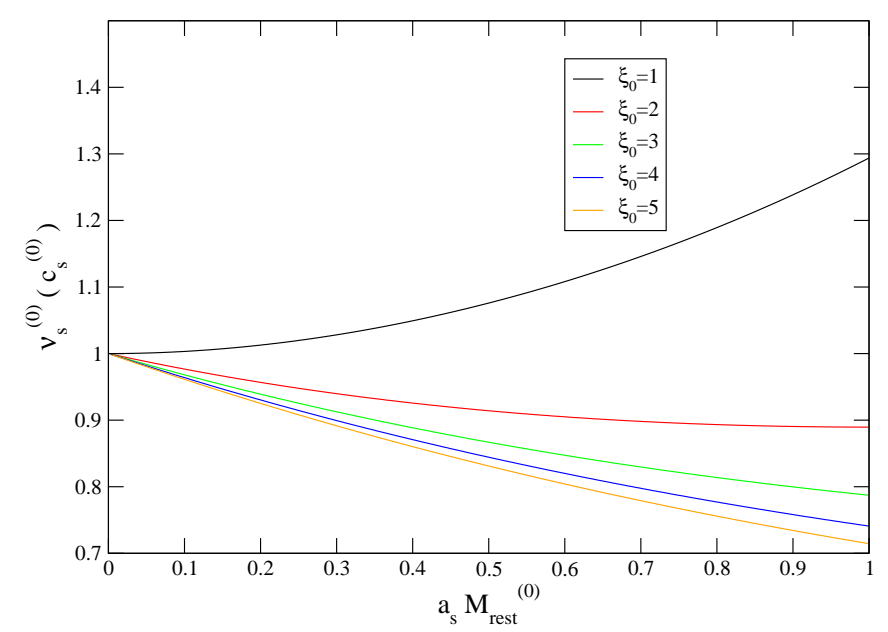

Figure 1: $v_{s}^{(0)}$ as a function of the tree-level quark rest mass given in spatial lattice units.

continuum counterparts. They are

$$
\begin{aligned}
c_{t}^{(0)} & =\frac{a_{t} m_{0}\left(2+a_{t} m_{0}\right)}{4 v_{s}^{(0)} \xi_{0}\left[\log \left(1+a_{t} m_{0}\right)\right]^{2}}-\frac{v_{s}^{(0)}}{\xi_{0} a_{t} m_{0}\left(2+a_{t} m_{0}\right)}, \\
c_{s}^{(0)} & =v_{s}^{(0)} .
\end{aligned}
$$

At tree level, our quark action is essentially a reparametrisation of the actions used in Refs. [5, 6], and accordingly Eq. 3.2 and Eq. 3.3 are in agreement with the results of those papers. Fig. 1 plots $v_{s}^{(0)}$ versus the tree-level rest mass for a number of bare anisotropy values. Note the quark mass dependence of $v_{s}^{(0)}$ even in the isotropic limit.

To go beyond tree level, we compute the quark energy and scattering amplitudes in perturbation theory and apply the same tuning criteria. Our calculation of the leading order correction to $v_{s}$ therefore amounts to a determination of the quark self-energy in one-loop perturbation theory.

\section{Gauge anisotropy}

The anisotropy parameter for the gauge action used in this study has previously been determined to one-loop order in pure Yang-Mills in Ref. [7]. In that study, twisted boundary conditions [8] were used as an infrared regulator for the gluons. The anisotropy was determined by demanding that one of the stable states in the twisted world, the so-called A meson, satisfy a relativistic dispersion relation. Determining the anisotropy, therefore amounts to a calculation of the one-loop gluon self-energy. We have repeated that calculation and extended it to include quark-loop effects. 


\section{Methodology}

Lattice perturbative calculations are notoriously complicated, even at the one-loop level. In our case, although only a moderate number of Feynman diagrams arise, the vertex functions which appear in these diagrams are extremely complicated due to smearing of the link variables in the quark action and Symanzik improvement of the gauge action. For such calculations, the best approach is to automate the derivation of vertex functions and the evaluation of the final momentum integrals as much as possible $[8,9,10]$.

To evaluate the vertex functions, we employed a number of independent methods. In one approach, we used the suite of Python code described in Ref. [10] to expand the actions to the required order in the coupling. We have written a parser which converts the resulting data file into a method of a corresponding C++ vertex class. As a cross-check, a completely separate suite of $\mathrm{C}++$ code has been developed which evaluates the momentum space vertex functions for a given set of four-momenta. Using this automated approach, we can evaluate vertex functions for an arbitrary level of link smearing. In fact, we have checked that it is possible to evaluate a four-gluon vertex function for the quark action with one hundred iterations of the stout-link smearing algorithm in just a few seconds.

Spin trace evaluations are completely automated, and the results presented here have been obtained using the Vegas integration routine [11]. Where derivatives of the self-energy with respect to external momentum are required, automatic differentiation can be applied directly to the integrand being passed to Vegas. However, the resulting sharply peaked function can prove difficult to integrate. In that case, more precise results may be obtained by evaluating the self-energy at different values of the external momentum and estimating the derivative numerically [12].

All calculations are performed in a Lorentz-covariant gauge and, where practicable, we repeat calculations in both Feynman and Landau gauge to verify the gauge-invariance of our results.

\section{Results}

A plot of the one-loop correction to $v_{s}$ as a function of the quark rest mass for a typical anisotropy value is shown in Fig. 2. The data presented here are coefficients of $g^{2}$. Two different levels of smearing are shown. The stout link smearing parameters of $n_{\rho}=2$ and $\rho=0.14$ were found to simultaneously minimise the additive quark mass renormalisation and maximise the spatial plaquette in one-loop perturbation theory [3]. For this choice of smearing parameters, the plot shows that tadpole improvement has little effect on $v_{s}$. In this study, as in our simulations, we define the tadpole factor to be the fourth root of the expectation value of the plaquette.

Regarding corrections to the gauge anisotropy parameter, there are a couple of points which are important to note. First, the pure gauge contribution to the anisotropy and the contribution from sea quarks are additive. That is to say, the correction for full QCD is simply the sum of the correction coming from pure Yang-Mills and the quark-loop contribution. Second, it is obvious that the magnitude of the correction coming from quark loops is proportional to $N_{f}$, so that it increases with an increasing number of flavours. It is also important to note that, at one-loop order, the seaquark contribution to the gauge anisotropy is independent of the choice of gauge action. Therefore, the quark-loop corrections presented here hold for any choice of anisotropic gauge action. 


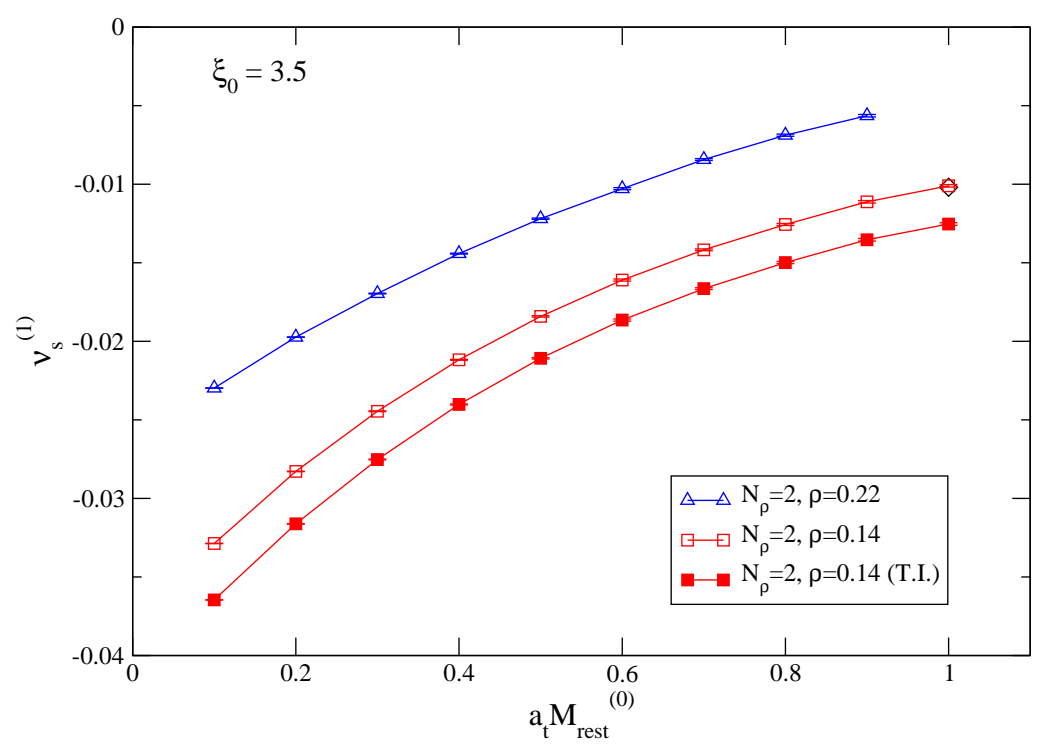

Figure 2: One-loop correction to $v_{s}$ as a function of quark mass. T.I. denotes tadpole improvement, while the hollow symbols denote results obtained before tadpole improvement.

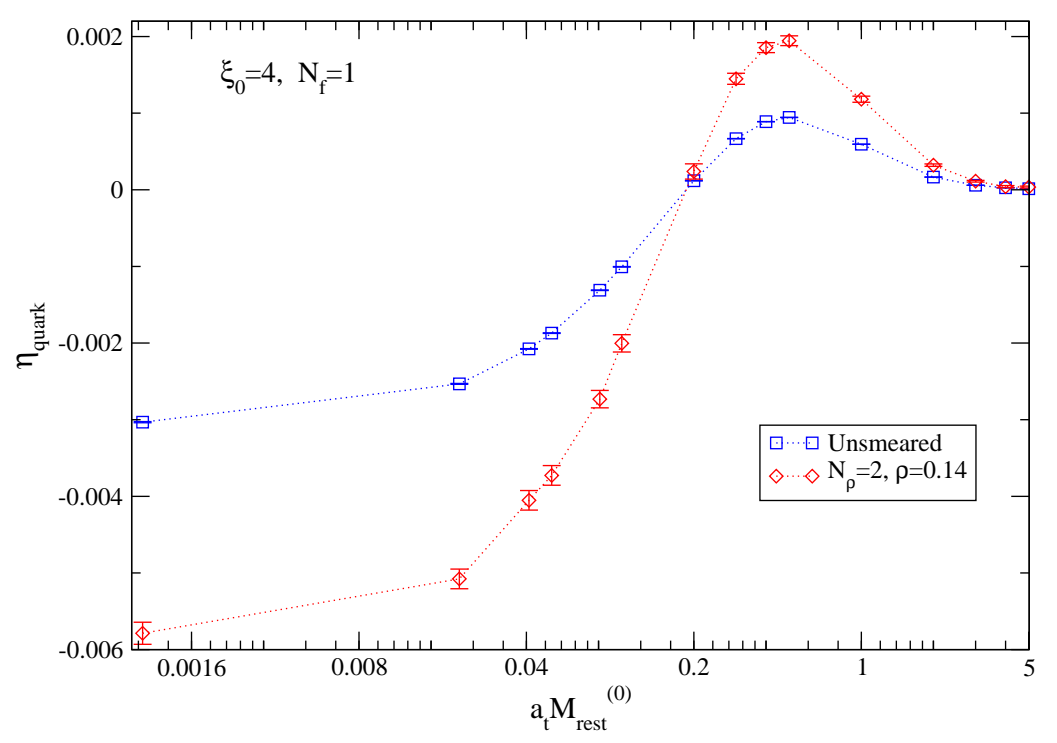

Figure 3: The quark-loop contribution to $\eta$ plotted as a function of the tree-level quark rest mass for different levels of smearing. As described in the text, $\eta$ is defined by $\xi_{g} / \xi_{0}=1+g^{2} \eta$, where $\xi_{g}$ denotes the anisotropy measured from the gluonic dispersion relation. 
Following Ref. [7], we define $\eta$ to be the one-loop correction to the anisotropy divided by the bare anisotropy value appearing in the gauge action. As a check on our methods, we calculated the pure gauge contribution to $\eta$ as described in that reference and found agreement with the results given there. The contribution of a single sea-quark flavour to this quantity for a fixed bare anisotropy but a varying sea-quark mass is shown in Fig. 3. As expected, this contribution goes to zero in the heavy quark limit, but becomes significant at light quark masses. At sufficiently light quark masses the contribution to $\eta$ from three degenerate quark flavours can match the purely gluonic contribution in magnitude [12].

\section{Conclusion and outlook}

Anisotropic lattice actions must be tuned to guarantee the restoration of Euclidean invariance in the continuum limit. We have described this tuning in one-loop perturbation theory. To remain as close to simulation as possible, the full quark mass dependence has been included in this calculation. Moreover, we have developed a suite of software capable of handling complicated actions involving an arbitrary level of link smearing. One-loop calculations of the quark action improvement coefficients $c_{s}$ and $c_{t}$ using this machinery are currently in progress.

Future work will obviously include a comparison with non-perturbative results. It will also be useful to know how the addition of an adjoint term to the gauge action, as outlined in Ref. [13], affects the tuning of the gauge anisotropy.

\section{Acknowledgments}

We are grateful to the authors of Ref. [10] for providing us with a copy of the 'HiPPy' Python code, which was used in the derivation of the vertex functions. This work was supported by the U.S. National Science Foundation under the Awards PHY-0510020 and PHY-0653315.

\section{References}

[1] C. Morningstar and M. Peardon, Phys. Rev. D56 4043-4061 (1997).

[2] C. Morningstar and M. Peardon, Phys. Rev. D60 034509 (1999).

[3] R.G. Edwards, B. Joo and H. W. Lin, arXiv:0803.3960.

[4] C. Morningstar and M. Peardon, Phys. Rev. D69 054501 (2004).

[5] A.X. El-Khadra, A.S. Kronfeld and P.B. Mackenzie, Phys. Rev. D55 3933-3957 (1997).

[6] J Harada, A.S. Kronfeld, H. Matsufuru, N. Nakajima and T. Onogi, Phys. Rev. D64 074501 (2001).

[7] I.T. Drummond, A. Hart, R.R. Horgan and L.C. Storoni, Phys. Rev. D66 094509 (2002).

[8] M. Lüscher and P. Weisz, Nucl. Phys. B266 309 (1986).

[9] C. Morningstar, Phys. Rev. D48 2265-2278 (1993).

[10] A. Hart, G.M. von Hippel, R.R. Horgan and L.C Storoni, J. Comput. Phys. 209 340-353 (2005).

[11] G.P. Lepage, J. Comput. Phys. 27192 (1978).

[12] J. Foley and C. Morningstar, in preparation.

[13] C. Morningstar and M. Peardon, Nucl. Phys. Proc. Suppl. 83 887-889 (2000). 